\title{
Representaciones sociales de personas transgénero travestis y transexuales) sobre la violencia
}

\author{
Luiz Paulo Ribeiro ${ }^{1}$, Sergio Rosa Neves Riani², Maria Isabel Antunes-Rocha ${ }^{3}$ \\ Universidade Federal de Minas Gerais
}

Este estudio analizó las representaciones sociales (RS) sobre la violencia para la población transgénero de Belo Horizonte, en la región Sudeste de Brasil. Fueron utilizadas entrevistas semiestructuradas y análisis de contenido mediante el software Iramuteq. Fueron entrevistadas nueve personas transgénero (travestis y transexuales) escogidos por disponibilidad. Los resultados apuntaron que los anclajes y objetivaciones de RS estudiada están organizadas en cuatro clases/categorías: (a) vivencias de violencia, (b) instituciones y proceso tradicional, (c) procesos identitarios y (d) cuerpo y vivencia social. Por lo tanto, los anclajes y objetivaciones de RS de la violencia están relacionados al proceso transicional y experiencias individuales y sociales a las cuales estos sujetos están sometidos, pudiendo estar relacionado a la aceptación y resistencia a la violencia.

Palabras clave: identidad de género, violencia, representaciones sociales, políticas públicas.

\section{Social representations of transgender people (transvestites and transexuals) about violence}

This study investigated the social representations about violence of the transgender population in Belo Horizonte, Minas Gerais, Southeastern Brazil. Semi-structured interviews and content analysis were used combined with lexical analysis through Iramuteq software. Nine transgender people (transvestites and transsexuals) were chosen for the interview based on convenience sampling. The results show that the anchorages and objectifications of social representations are organized into four categories: (a) experiences of violence (b) institutions and traditional process, (c) identity processes (d) and body and social life. Consequently,

1 Doctor en Educación. Profesor adjunto A en el Departamento de Ciencias Aplicadas a la Educación en la Facultad de Educación de la Universidad Federal de Minas Gerais. Dirección postal: Facultad de Educación, oficina 1619, de la Universidad Federal de Minas Gerais, Av Antônio Carlos, 6627 - Pampulha - Belo Horizonte - MG. Contacto: luizpr@ufmg.br https://orcid.org/0000-0002-4278-7871

2 Magíster en Criminología Crítica, Seguridad Pública y Prevención Social por la Universidad de los Estudios de Padova-Italia. Magíster en Promoción a la Salud y Prevención de la Violencia en la Facultad de Medicina (UFMG). Coordinador del Centro de Referencia Especializado de Asistencia Social del Ayuntamiento de Belo Horizonte - Minas Gerais. Contacto: sergioriani@gmail.com https://orcid.org/0000-0002-7429-594

3 Doctora en Educación. Profesora asociada en el Departamento de Ciencias Aplicadas a la Educación en la Facultad de Educación de la Universidad Federal de Minas Dirección postal: Facultad de Educación, oficina 1619, de la Universidad Federal de Minas Gerais. Av Antônio Carlos, 6627 - Pampulha - Belo Horizonte - MG. Contacto: isabelantunes@fae.ufmg.br https://orcid.org/0000-0002-4044-6723 
the anchorages and objectification of the social representationsof violence are related to the transitional process, the individual and social experiences to which these people are subjected that could be related to the acceptance and resistance to violence.

Keywords: gender identity, violence, social representations, public policy.

Representaçóes sociais de pessoas transgêneras (travestis e transexuais) sobre a violência Este estudo analisou as representaçóes sociais sobre a violência para a populaçáo transgênera de Belo Horizonte, Minas Gerais, na regiáo Sudeste do Brasil. Foram utilizadas entrevistas semiestruturadas e análise de conteúdo conjugada com análise léxica através do software Iramuteq. Foram entrevistadas 09 (nove) pessoas transgêneras (travestis e transexuais) escolhidas por disponibilidade. Os resultados apontaram que as ancoragens e objetivaçôes das RS estudadas estão organizadas em quatro classes/categorias: (a) vivências da violência (b) instituiçóes e processo transicional, (c) processos identitários e (d) corpo e vivência social. Assim, as ancoragens e objetivaçóes da RS da violência está relacionada ao processo transicional e experiências individuais e sociais às quais estes sujeitos estão submetidos que podem estar relacionados à aceitação e resistência à violência.

Palavras-chave: identidade de gênero, violência, representaçôes sociais, políticas públicas.

\section{Représentations sociales des personnes transgenres (travestis et transsexuels) à propos de la violence}

Cette étude a analysé les représentations sociales (RS) sur la violence au sein de la population transgenre de Belo Horizonte, dans la région du sud-est du Brésil. Des entretiens semistructurés et l'analyse de contenu ont été utilisés via le logiciel Iramuteq. Neuf personnes transgenres (travestis et transsexuels) choisies pour la disponibilité ont été interviewées. Les résultats ont montré que les ancres et les objectifications de RS étudiées sont organisées en quatre classes / catégories: (a) expériences de violence, (b) institutions et processus traditionnels, (c) processus identitaires et (d) expériences corporelles et sociales. Par conséquent, les ancrages et les objectifications de la violence dans la RS sont liés au processus traditionnel et aux expériences individuelles et sociales auxquelles ces sujets sont soumis, et peuvent être liés à l'acceptation et à la résistance à la violence.

Mots-clés: identité de genre, violence, représentations sociales, politiques publiques. 
La Teoría de las Representaciones Sociales fue iniciada por la tesis de doctorado de Serge Moscovici en 1962. En esa obra, la iniciativa fue reconocer cómo saberes académico-científicos (universo reificado) eran absorbidos y relaborados en el sentido común (universo consensual), tomando como referencia la dispersión del psicoanálisis en Francia (Moscovici, 2012). A lo largo de más de 50 años de investigación y evoluciones teóricas en este campo de estudio, esta teoría se expandió y fue aplicada en diferentes contextos, sujetos y objetos. Esa expansión, como apunta Jodelet (2001), se dio por la posibilidad de entender cómo la subjetividad y la sociabilidad son recíprocas en la estructuración de cognición social. En este sentido, se entiende que el pensar, el sentir y el actuar están relacionados a los procesos de aprehensión de los objetos socialmente compartidos, pudiendo sufrir influencia del contexto, de las actitudes y de las informaciones que circulan en la sociedad (Ribeiro \& Antunes-Rocha, 2016).

Se sabe que el proceso de construcción de una representación social genera el cambio de lo que es extraño en familiar; en otras palabras, un movimiento de asimilación socio cognitiva (objetivación y anclaje) en que determinados objetos se vuelven agradables a la vivencia social. En este proceso puede haber formación de preconceptos y estigmatización, una vez que la propia sociedad lee y organiza sus contenidos y saberes a partir de una matriz de análisis que es histórica, material y socialmente compartida. De este proceso no escapan sujetos ni objetos, una vez que hay una triada de representación sujeto-objeto-otro, donde hay una reciprocidad de representaciones (Jodelet, 2001).

Se rescata que la Teoría de las Representaciones Sociales (TRS) tiene su origen en la psicología social y en la psicosociología y que expresa "[...] conjuntos dinámicos [...] la producción de comportamientos y de relación con el ambiente, de la acción que modifica unos y otros [...]" (Moscovici, 2012, p. 47), o sea envuelven la búsqueda del 
entendimiento sobre la dinámica individual y social la cual moviliza a los sujetos a sentir, pensar y actuar. La representación social es algo vivo, que está imbricado con la intención y la interacción entre el sujeto y la sociedad, en una relación intensa, de ir y venir, en la cual tanto el sujeto como la sociedad producen y reproducen conceptos, símbolos e imágenes (Moscovici, 2012).

De forma simple, una representación social puede estar organizada a partir de objetivaciones y anclajes (Moscovici, 2012). objetivación se entiende como una tentativa de dar sentido al objeto representado. Se puede definir como anclaje el proceso por el cual el objeto representado es elegido como esquema referencial preexistente, dando sentido al mismo.

En este sentido, la violencia que es fatídica y vivencial en diferentes culturas y contextos es un objeto representable que demarca sujetos, conductas y afectividades (Ribeiro, 2017). Así, Porto (1999) trae a la atención nuevos patrones de socialización que han sido construidos "[...] en la y por la violencia, casi como respuesta a la carencia, ausencia, fallas y rupturas" (p. 131). En esta dirección, la violencia es erigida como un recurso, una posibilidad para la acción. Porto (1999) dice que para las populaciones de baja y media renta hay representaciones sociales sobre los ricos como más violentos. También se observa que hay una relación recíproca: en este caso, es común la consideración por los más ricos que las capas menos favorecidas de la población también sean consideradas violentas. Para Porto (1999), la violencia es un fenómeno articulado con la realidad de los sujetos y sociedades, marcado por la experiencia y caracterizado como un contenido para disputar la hegemonía. Esta autora vincula también la violencia a una concepción identitaria de sí, o que erige la identificación a partir de una conducta, algo posible de verificar inclusive en dichos populares, como: "homem que é homem não leva desaforo para casa ${ }^{4 ”}$ (p. 262).

4 Se decidió mantener la frase en portugués ya que es un dicho brasileño y podríamos perder su significado. 
El estudio de Guimarães y Campos (2007) sobre las representaciones sociales de la violencia en adolescentes, estudiantes de la Enseñanza Media de Goiânia - Goiás, encontró dos tipos: vinculada a la noción o la representación de violencia como acto agresivo(prioritariamente, acto agresivo resultante en muerte) y; la naturalización o banalización de la violencia el debido al involucramiento directo de los sujetos con la violencia. Sobre esta última, ellos traen a la atención sobre: Lo que caracteriza fundamentalmente la noción de banalización de la violencia es la legitimación del uso de la agresión (física o simbólica) como forma de regulación o resolución de conflictos de intereses, sea entre personas o grupos. Guimarães y Campos (2007) también resaltan a la representación social de la violencia como una caraterística de lo cotidiano, algo que viene de una disposición cultural derivada de un rompimiento de acuerdos y negociaciones y, la tendencia resolver situaciones a partir de agresiones físicas y verbales.

Oliveira, Chamon y Maurício (2010) estudiaron la RS de la violencia en estudiantes de diferentes promociones en una universidad en el interior del estado de São Paulo. Fueron entrevistados 20 individuos y los datos fueron analizados a partir del software Alceste. Los participantes de la investigación representan la violencia como consecuencia del desamparo estatal en el sistema capitalista y de las desigualdades sociales; la falta de amor, una desorganización de la vida familiar y la violencia doméstica resultante de estos también son engendradas en la RS de violencia.

Cortez, Menandro y Souza (2010) realizaron un minucioso estudio de las representaciones sociales sobre la violencia de género y violencia conyugal presentes en estudios científicos. Ellos analizaron 280 producciones científicas y notaron que: (i) la mujer está socialmente más vulnerable a la violencia en las relaciones afectivas; (ii) las relaciones desiguales de poder relación con una base patriarcal y machista son potencialmente violentas; (iii) que la calidad de atención en los servicios de salud depende de las percepciones que los profesionales tienen de la violencia y, (iv) la violencia aparece indistintamente etiquetada como una característica masculina. 
El artículo de Assis (2013) trata las representaciones sociales sobre la violencia de género en adolescentes del municipio de Cacoal en el estado de Roraima. Las representaciones sociales de la violencia de género de estos sujetos apuntan a que la violencia de género está vinculada a la falta de comunicación, al uso abusivo de alcohol y otras drogas y al machismo. Para los sujetos estudiados, para resolver la violencia es necesario un mayor acceso a la educación, mejoras gubernamentales y la voluntad individual. Para la autora, los resultados encontrados remiten a la peligrosidad de considerar a veces a la violencia como factor que debe ser resuelto internamente.

Correia (2014) hace un estudio sobre las RS del suicidio, en mujeres con historial de violencia doméstica y con intento de suicidio. Fueron 20 sujetos de investigación sometidos a la Prueba de Asociación Libre Palabras En este estudio, apuntaron que "la representación de las mujeres sobre el suicidio se encuentra anclada en historias de vida permeada por el rechazo y el desamor, que llevan a la enfermedad, sobre todo a la depresión. El sentimiento de impotencia ante la necesidad de cambio y liberación desencadena problemas de alcance emocional que culminan en la decisión de interrumpir la propia vida” (p. 118).

A partir de esos relatos de investigación sobre las RS de la violencia para distintos sujetos se encontró que: (i) el área que se preocupa por los estudios sobre la violencia es el área de salud, ya que la violencia es encuadrada como causas externas que son evitables y no transmisibles; (ii) las RS aquí enumeradas hablan de sujetos que se involucran o no en situaciones de violencia, o que están potencialmente expuestos en un contexto en que la violencia existe, en los que son víctimas; (iii) la RS de la violencia es compleja, ya que se relaciona con otras como las de seguridad pública, policía, crimen, salud y, principalmente, justicia y sujeto de derecho; (iv) por ser un tema que causa miedo, intimidación y vergüenza en muchos sujetos, aun cuando hayan sido víctimas de la violencia, tienden a hablar del sufrimiento de otras personas, colocándola lejos de sí, o no reconociéndose como víctimas. Estas investigaciones ayudan a entender lo que puede ser concebido como violencia junto a la población travesti y transexual en el contexto estudiado. 


\section{Transexualidad y violencia}

Se parte del supuesto que la violencia, según Ribeiro (2017) es un fenómeno social que puede ser notado en diferentes sociedades y culturas, así como, tiene relación con las formas de ejercer el poder y de los padrones de sociabilidad, además de que en una lógica sociabilizada, los actos de violencia pueden ser justificados por las prácticas culturales instaladas y que no hay como "vivenciar la violencia sin la producción de algo" (p. 36). Es decir, la violencia no es algo restringido a la población travesti y transexual, pero por existir una cultura que todavía es machista, patriarcal y cisheteronormativa ella termina siendo permitida y muy motivada por el odio y la LGBTfobia. Sin embargo, por ser producto social demuestra que es posible de modificación de su status quo, o sea, incluso frente a la verificación de un extenso rastro de violencia contra la población transgénero, es posible tener esperanza de superación a partir de la divulgación, concientización, protagonismo $\mathrm{y}$ reconocimiento de las diferentes formas de identidad de género y práctica sexual.

Aunque la cuestión de la transexualidad ocupe los espacios de medios sociales y televisivos en Brasil (Itaqui \& Felippi, 2017), lo que se ha observado es que Brasil continua, paradójicamente, siendo el país que más mata transexuales y travestis en el mundo (Transgender Europe, 2017), como también es el país donde los índices de búsqueda por contenido pornográfico con personas transgénero son los más visitados (Rocha, Ban \& Valenga, 2017). Esa lógica del uso y de cultura de la violencia contra las personas transgénero (travestis y transexuales) refleja la vivencia social y las representaciones sociales sobre estos sujetos que están en la marginalidad y extrema vulnerabilidad y acaban por encontrar en la prostitución una única salida para el enfrentamiento de sus fragilidades. Hay que reflexionar sobre tales representaciones y vivencias, ya que en la prostitución los sujetos transgéneros son usados como fetiches sexuales, objetos de placer y sujetos desechables, ya que no son raros los casos de asesinato de personas transexuales y travestis después de la realización de 'programas'. 
Sin embargo, aunque sea una situación que despierta la tristeza y la indignación, la situación de violencia contra las personas transgénero va más allá de la cuestión de la prostitución. Es muchas veces, en la familia, en el proceso transición que esos sujetos experimentan gran parte de la violencia: rechazo, expulsión de casa, humillaciones y culpabilizaciones, etc., lo que hacen que estos sujetos rompan sus vínculos familiares muy temprano, siendo bajos en las capas más populares los registros de aceptación de la familia sobre la transexualidad de los hijos (Efrem Filho, 2016). Se entiende que las formas con que estos sujetos son vistos (como son las representaciones sociales construidas sobre ellos), son de cierta extrañeza y marcado con preconceptos y subyugación, lo cual contribuye a este status quo violento y expropiador.

Por definición, se dice que transgénero es un término 'paragua' utilizado para conceptuar y describir a aquellas personas cuya identidad y/o la expresión de género difiere, incumple, viola, hiere, confronta, es conflictiva o no está en conformidad con el binarismo de género sostenido en las normas sociales referentes al sexo que determinado individuo fue asignado al nacer (Lanz, 2014). Estos individuos género-divergentes hacen parte de lo que Lanz (2014) conceptualiza como 'transgeneridad' que es un fenómeno sociológico que desvía o transgrede el dispositivo binario de género, abarcando las identidades género-divergentes tales como transexuales, travestis, crossdressers, dragqueens, andróginos, etc.

La vivencia en la escuela y en el acceso a otros servicios públicos, como el de salud, no quedan fuera del conjunto de violencias que las personas transgéneros sufren. En la escuela, además del bullying y de la transfobia sufridos cotidianamente por parte de los demás alumnos, las personas transgéneros encuentran problemas en la utilización de las instalaciones de la escuela, como el vestuario y el baño (Cruz, 2011; Efrem Filho, 2016). Por otro lado, algunas veces, los profesionales de la escuela, que deberían promover un lugar de protagonismo escolar y de convivencia con las diferencias, acaban haciendo lo contrario: auxilian en la cultura de la estigmatización y no promueven el diálogo y el respeto. Esas conductas influyen en la baja escolaridad de las personas trans, una vez que no siendo aceptado en el ambiente escolar, 
abandonan la escuela, incidiendo directamente en la actuación profesional de esos sujetos (Souza \& Bernardo, 2014).

En el sistema de salud, el preconcepto de los profesionales incide directamente con la calidad de la atención de las personas transgéneros, provocando deshumanización y no atención a sus necesidades (Silva, et al., 2016). La violencia parte desde el no respeto al uso del nombre social, hasta acciones vejatorias y discriminatorias durante la atención; además de conductas que la diagnostican como enfermedad (Arán, Murta, \& Lionço, 2009). Aunque existe el "proceso transexualizador del SUS” (Brasil, 2013), una orientación de la política pública para que las personas transgéneros sean acogidas y respetadas en su individualidad y en su proceso de salud-enfermedad, todavía hay barreras en su implantación y expansión en todos los equipos de atención básica y especializada en salud. Sin embargo, se enfatiza que esta orientación, que también se convirtió en una legislación, es un avance antihegemónico.

Si estos sujetos no tienen apoyo familiar, acaban por encontrar refugio en la prostitución, ingresos y una inclusión social disfuncional. Si en la política pública de salud no se tiene atención a sus necesidades, recurren a instituciones clandestinas y al uso de materiales inapropiados para obtener resultados en relación al uso de hormonas y procedimientos estéticos en la adecuación del cuerpo a la identidad de género (Ventura, 2010). En la escuela aún enfrentan transfobia y preconcepto y esas situaciones inciden directamente en su inserción en el mercado de trabajo - la informalidad y la baja cualificación profesional (Zanin, Ferreira, \& Ribeiro, 2019), además de la necesidad de a veces tener que omitir al máximo su transexualidad para mantenerse en el trabajo (Carrieri, Souza \& Aguiar, 2014) — además de influir en su expectativa de vida y prácticas sociales vulnerables. Estas situaciones, por sí solas, son síntomas y desencadenan en un ciclo de la violencia que persiste en la población transgénero en Brasil.

Es decir, la población travesti y transexual encuentra diferentes manifestaciones de violencia en su cotidiano pero, ¿qué es lo que esos sujetos piensan, sienten y cómo actúan ante ese fenómeno? ¿Cómo ellos la vivencian y cuáles son sus propuestas para la mejora del cuadro social 
y el alcance de derechos sociales ya conquistados? Así, la propuesta de este artículo es analizar, las representaciones sociales de la violencia que tienen sujetos transgéneros (específicamente transexuales y travestis) de Belo Horizonte, Brasil.

Por otro lado, utilizar la teoría de las representaciones sociales significa entrar en contacto con la vivencia de la violencia por parte de las personas transgéneros y de cómo las formas de pensar, sentir y actuar de esos sujetos son movilizadas ante ese fenómeno (Moscovici, 2012). Por su parte, Jodelet (2005), evidenció que las experiencias vividas contribuyen significativamente a la construcción social-subjetiva de la realidad, o sea, "el sistema global de representaciones proporciona los recursos y los instrumentos para interpretar aquello que es experimentado [al paso que] esa experiencia da sentido a lo vivido que estructura" (Jodelet, 2005, p. 48).

A pesar de los estudios que involucran las RS y la transexualidad/ travesti ya sean comunes (Santos, Shimizu \& Merchan-Hamann, 2014; Baptista, Silva, Menandro \& Bonomo, 2016; Matão, Miranda, Campos, Teles \& Mesquita, 2010; Arbatani, Aqili, Labafi \& Omidi, 2016); aún son escasos los estudios que incluyen tales sujetos como participantes (Caravaca-Morera \& Padilha, 2017), existiendo un espacio provechoso para este campo de investigación.

\section{Método}

Esta es una investigación cualitativa-descriptiva con sujetos travestis y transexuales del municipio de Belo Horizonte, en el estado de Minas Gerais, Brasil. Es una investigación dentro del paradigma de las Teorías de las RS (Moscovici, 2012; Jodelet, 2005). Esta producción se inició de una investigación mayor que buscaba entender las vivencias de las personas transgéneros en el acceso y utilización de los servicios de salud, educación y asistencia social de la ciudad capital del estado de Minas Gerais. Por ser uno de los asuntos notados como relevantes se decidió investigar las RS de la violencia a fin de verificar sus relaciones con la investigación mayor. 
Para ello fueron realizadas nueve entrevistas semiestructuradas con sujetos transgénero que se ofrecían a participar voluntariamente de la investigación. Al inicio, se preveía que la muestra sería de aproximadamente 30 sujetos, pero los investigadores encontraron dificultades en el proceso de participación, debido al extenso rastro de violencia y aumento de las investigaciones sin retorno a los mismos, estos sujetos se han rehusado a participar en estudios que los involucran. La Tabla 1 traza el perfil socioeconómico de los participantes de la investigación. El criterio identificación fue hecho por auto denominación, o sea, los participantes se les preguntó por su identidad de género, lo cual fue respetado.

\section{Tabla 1}

Datos demográficos de los participantes

\begin{tabular}{|c|c|c|c|c|c|}
\hline $\mathrm{n}$ & Edad & $\begin{array}{l}\text { Identidad de } \\
\text { género }\end{array}$ & $\begin{array}{c}\text { Nivel de } \\
\text { escolaridad }\end{array}$ & $\begin{array}{c}\text { Renta } \\
\text { mensual }\end{array}$ & $\begin{array}{l}\text { Vínculo de } \\
\text { trabajo }\end{array}$ \\
\hline A & 54 & Mujer transexual & $\begin{array}{l}\text { Enseñanza media } \\
\text { completo }\end{array}$ & $\begin{array}{l}\text { Más de } 5 \text { salarios } \\
\text { mínimos }\end{array}$ & $\begin{array}{l}\text { Trabajadora sexual } \\
\text { Profesora }\end{array}$ \\
\hline B & 30 & Mujer transexual & Técnico & $\begin{array}{l}\text { Hasta } 5 \text { salarios } \\
\text { mínimos }\end{array}$ & Peluquera \\
\hline C & 33 & Mujer transexual & $\begin{array}{l}\text { Enseñanza media } \\
\text { completo }\end{array}$ & $\begin{array}{l}\text { Más de } 5 \text { salarios } \\
\text { mínimos }\end{array}$ & $\begin{array}{l}\text { Profesional del } \\
\text { sexo }\end{array}$ \\
\hline $\mathrm{D}$ & 24 & Mujer transexual & $\begin{array}{l}\text { Enseñanza media } \\
\text { completo }\end{array}$ & $\begin{array}{l}\text { Menos de } 1 \\
\text { salario mínimo }\end{array}$ & No tiene \\
\hline $\mathrm{E}$ & 23 & Mujer transexual & Técnico & $\begin{array}{l}\text { Menos de } 1 \\
\text { salario mínimo }\end{array}$ & Modelo \\
\hline $\mathrm{F}$ & 34 & Mujer transexual & $\begin{array}{l}\text { Enseñanza media } \\
\text { incompleto }\end{array}$ & $\begin{array}{l}\text { Un salario } \\
\text { mínimo }\end{array}$ & $\begin{array}{l}\text { Profesional del } \\
\text { sexo }\end{array}$ \\
\hline G & 26 & $\begin{array}{l}\text { Travesti } \\
\text { transfemenina } \\
\text { no-binaria }\end{array}$ & $\begin{array}{l}\text { Enseñanza media } \\
\text { completo }\end{array}$ & $\begin{array}{l}\text { Un salario } \\
\text { mínimo }\end{array}$ & Autónoma \\
\hline $\mathrm{H}$ & 41 & Mujer transexual & $\begin{array}{l}\text { Enseñanza } \\
\text { Superior }\end{array}$ & $\begin{array}{l}\text { De } 2 \text { a } 5 \text { salarios } \\
\text { mínimos }\end{array}$ & Contratado \\
\hline I & 26 & $\begin{array}{l}\text { Hombre } \\
\text { transexual } \\
\text { no-binario }\end{array}$ & $\begin{array}{l}\text { Enseñanza media } \\
\text { completo }\end{array}$ & $\begin{array}{l}\text { Menos de un } \\
\text { salario mínimo }\end{array}$ & Desempleada \\
\hline
\end{tabular}


Por su parte, el protocolo de preguntas que fue utilizado en las entrevistas fue construido a partir de presupuestos de la teoría utilizada, con preguntas que posibilitaran describir la experiencia de los sujetos ante el objeto violencia, analizarlo y prescribir acciones ante lo mismo. Por otro lado, también se atendió a las dimensiones de la representación social, en la construcción de las preguntas buscando identificar fuentes de información, elementos contextuales y actitud de los sujetos (Ribeiro \& Antunes-Rocha, 2016). La Tabla 2 trae la organización de preguntas por temática del rutero de entrevista aplicado.

\section{Tabla 2}

\section{Guia de entrevista}

\begin{tabular}{|c|c|}
\hline Tipo de 1 & Preguntas \\
\hline Definición & $\begin{array}{l}\text { ¿Qué es ser TRANS? ¿Cómo usted se asumió como una } \\
\text { persona trans? ¿Cuáles son los impactos de eso para a su } \\
\text { vida? ¿Cuáles cambios hubo en su vida? ¿Cómo fue la } \\
\text { aceptación de la familia? }\end{array}$ \\
\hline Transgenitalización & $\begin{array}{l}\text { ¿Usted tiene interés en la transgenitalización? ¿Cómo } \\
\text { pretende/hizo eso? ¿O que usted piensa sobre eso? }\end{array}$ \\
\hline $\begin{array}{l}\text { Referencia técnica del } \\
\text { profesional }\end{array}$ & $\begin{array}{l}\text { ¿Cómo fue a su vivencia en la escuela, en el acceso a } \\
\text { la salud? ¿Hubo alguna violencia por parte de los } \\
\text { profesionales? ¿Cómo usted orientaría los profesionales } \\
\text { de esas áreas para mejor atención de la población } \\
\text { TRANS de Belo Horizonte? }\end{array}$ \\
\hline Vivencia del profesional & $\begin{array}{l}\text { ¿Al utilizar las políticas públicas, como fue su contacto } \\
\text { con los profesionales? ¿Hubo violencias? }\end{array}$ \\
\hline la violencia & $\begin{array}{l}\text { ¿A qué tipo de violencias las personas trans están sujetas? } \\
\text { ¿Cómo a X (educación, salud o asistencia social) ha } \\
\text { dado cuenta de eso? }\end{array}$ \\
\hline $\begin{array}{l}\text { Acceso a las políicas } \\
\text { públicas }\end{array}$ & $\begin{array}{l}\text { ¿Cómo es el acceso de la población a las políticas } \\
\text { públicas? ¿Encontraron barreras? ¿Son bien atendidos? } \\
\text { ¿Existe violencia en el atendimiento? ¿Sus demandas son } \\
\text { ovidas y derechos garantizados? }\end{array}$ \\
\hline $\begin{array}{l}\text { Atendimiento integral } \\
\text { no violento }\end{array}$ & $\begin{array}{l}\text { ¿Cómo las personas trans deberían ser atendidas en las } \\
\text { políticas públicas (educación, salud e asistencia social)? }\end{array}$ \\
\hline
\end{tabular}


Las entrevistas fueron recolectadas entre los meses de junio y octubre del año 2017. Todos los sujetos, resguardando los patrones éticos en investigación, fueron sometidos al término de consentimiento libre y esclarecido (TCLE). Sus conversaciones fueron transcritas y sus identidades fueron preservadas, retirando todos los elementos que permitieran tal identificación. En este artículo los participantes serán referidos solo por letras.

Después de las entrevistas hechas y los datos transcritos fue hecha una lectura atenta de todos los datos textual verificando posibles temáticas presentes en las conversaciones de los participantes. Los datos categorizados fueron tratados a través de análisis de contenido por categorías conjugada con el análisis léxico (Camargo \& Justo, 2013) con ayuda del software IRAMUTEQ.

\section{Análisis de datos}

El material analizado por la interfaz del IRAMUTEQ fue compuesto por 9 entrevistas y fue subdividido en 795 unidades de contexto elemental (UCE). A partir de estos caminos fue posible obtener correlaciones entre las palabras

\section{Resultados y discusión}

La interfaz del IRAMUTEQ reorganizó todo el corpus en cuatro clases a partir de la Clasificación Jerárquica Descendiente (CHD), dando posibilidad al dendograma presente en la figura 1. A saber, conforme Camargo y Justo (2013):

O método da Classificação Hierárquica Descendente (CHD) proposto por Reinert (1990) e utilizado pelo software ALCESTE classifica os segmentos de texto em funçáo dos seus respectivos vocabulários, e o conjunto deles é repartido com base na frequência das formas reduzidas (palavras já lematizadas). Esta análise visa obter classes de UCE que, ao mesmo tempo, apresentam vocabulário semelhante entre si, e vocabulário diferente das UCE das outras classes (p. 516). 


\begin{tabular}{|c|c|c|c|c|c|c|c|}
\hline \multirow{2}{*}{\multicolumn{2}{|c|}{$\begin{array}{c}\text { Instituciones } \\
\text { y proceso } \\
\text { transicional } \\
32 \% \text { de los datos }\end{array}$}} & \multicolumn{2}{|c|}{$\begin{array}{c}\text { Vivencias de la } \\
\text { violencia }\end{array}$} & \multicolumn{2}{|c|}{$\begin{array}{c}\text { Identidad y } \\
\text { género }\end{array}$} & \multicolumn{2}{|c|}{$\begin{array}{c}\text { Cuerpo y } \\
\text { vivencia social }\end{array}$} \\
\hline & & \multicolumn{2}{|c|}{$25 \%$ de los datos } & \multicolumn{2}{|c|}{$14.8 \%$ de los datos } & \multicolumn{2}{|c|}{$28.2 \%$ de los datos } \\
\hline Expresión & $\mathrm{X}^{2}$ & Expresión & $\mathrm{X}^{2}$ & Expresión & $\mathrm{X}^{2}$ & Expresión & $\mathrm{X}^{2}$ \\
\hline hoy & 48.61 & Así & 46.17 & hombre & 93.09 & travesti & 46.52 \\
\hline año & 47.44 & Nombre & 39.69 & cisgénero & 58.63 & cuerpo & 25.73 \\
\hline escuela & 39.75 & Cuando & 31.75 & mujer trans & 51.55 & nada & 25.42 \\
\hline familia & 25.99 & Notar & 30.57 & cis & 46.75 & sociedad & 24.74 \\
\hline siempre & 25.24 & Llegar & 29.82 & hombre trans & 40.84 & morir & 20.79 \\
\hline madre & 23.78 & Llamar & 23.2 & identificar & 38.35 & gente & 18.56 \\
\hline bueno & 22.76 & Respeto & 22.27 & mujer & 38.21 & $\mathrm{mal}$ & 15.87 \\
\hline sentir & 22.44 & Falta & 21.99 & parar & 28.29 & visibilidad & 14.05 \\
\hline padre & 21.59 & incentivo & 21.29 & impuesto & 24.62 & harmonio & 13.3 \\
\hline asumir & 19.84 & preparar & 19.93 & diferente & 16.51 & programa & 12.81 \\
\hline
\end{tabular}

Figura 1. Dendograma de las representaciones sociales sobre la violencia para sujetos transgéneros de Belo Horizonte $(n=9)$.

\section{Instituciones y proceso tradicional}

Esta clase fue constituida por el 32\% de los datos, siendo la mayor de las clases. Ella tiene tanto elementos de la vivencia en familia y escuela, como de la transición (reconocerse, vivir y asumir la transgeneridad $\left.^{5}\right)-\mathrm{y}$ cómo las instituciones ya enumeradas participaron de ese proceso-. Las palabras 'hoy, 'año' , 'escuela', 'mucho', 'familia', 'siempre', 'madre', 'bueno', 'sentir', 'padre', entre otras son las más frecuentes en esa clase.

La representación social de la violencia pasa por su vivencia histórica, al proceso de constitución de una identidad y de la personificación de ese sujeto. Así, ser transexual la palabra 'hoy' hace referencia a la transición y de lo que se sufrió en la familia y en la escuela hasta llegar

5 La transgeneridad se relaciona a la identidad de género, de ser, se asumir y se identificar como sujeto/a transgênero/a. 
a la etapa actual, como se evalúa en el presente. Ribeiro (2017) al analizar las RS de la violencia entiende que tales representaciones son construidas a lo largo de las vivencias de los sujetos de sus inserciones sociales y en las instituciones, siendo que no es posible pasar por la violencia sin representarla.

La apropiación de la identidad transgénero, asumir para sí y para los demás, forma parte de un proceso de aceptación que puede o no contener rasgos o percepciones de violencia. En ese recorrido, la utilización de la palabra 'año' es instrumento de demarcación de los espacios de tiempo, ante las experiencias.

Olha, a aceitação não é fácil. A aceitação da família. Primeiramente a família, no começo ela não acredita, ela acha que isso é loucura. A minha mãe mesmo, quando, eu mesmo não querendo, eu nunca precisei de assumir porque estava a olhos vistos (Entrevistada B, Mulher Transexual).

[¿Los amigos te aceptan?] Los amigos sí, porque así que yo me asumí trans poco después de 1 año yo adopté mi nombre, hoy en día [...] todo el mundo ya me conoce (Entrevistada H, Mujer Transexual).

Yo estuve muchos ańos en Europa, no tuve ganas de venir para Brasil porque allí la gente es tratada como una mujer normal, entonces no existe este preconcepto (Entrevistada A, mujer transexual).

El proceso de asumir fue muy tranquilo porque ya me había identificado como transexual hace más de cuatro ańos. Solo que investigué bastante porque sabía que en cualquier momento alguien iba a intentar deslegitimar lo que estaba hablando que eu era. Yo salí de una cajita que me tenía atrapado desde hace muchos años [...] de alguna manera perjudicó mucho mi vida (Entrevistada G, travesti, transfeminina, no binaria).

La temporalidad, indicada por la secuencia de hechos historizados, también aparece como un demarcador de las relaciones entre el sujeto y el objeto en la constitución de las representaciones sociales. Jodelet (2005) al hablar de las experiencias de los sujetos y la formación de representaciones sociales apunta a la composición de fenómenos que 
se van constituyendo a partir de la aproximación y la vivencia de los sujetos. En una vertiente temporalizada, al acercarse de los objetos los sujetos son presionados a inferir sobre ellos —elaborar representaciones sociales - en que los sujetos pueden negar a vivenciar lo nuevo, asimilar totalmente lo nuevo, o aún, promover un status de mantenimiento de lo que se tenía con lo que se tiene como nuevo, emparejando (AntunesRocha, Amorim-Silva, Benfica, Carvalho \& Ribeiro, 2015; Ribeiro, Carvalho \& Antunes-Rocha, 2017).

Ante las experiencias y sus temporalidades, la familia, sus parientes, la escuela y los profesionales asumen un papel destacado en esta clase, ya que son estas instituciones que promueven las primeras socializaciones de la sexualidad y es en ellas que las primeras situaciones de violencia también son percibidas. Sin embargo, el descubrimiento de la transexualidad en la infancia y en la escuela es evaluada como más difícil ya que encuentra barreras en lugares que deberían ser promotores de la libertad y de la aceptación.

Pero creo que si hubiera descubierto mi transición en el período de la escuela, creo que sería mucho más difícil y es más difícil para la gente que se descubre en el período escolar tanto en la infancia (Entrevistado D, hombre transexual).

Fui prohibida de asistir a la escuela, dijeron que podía ser una mala influencia para los alumnos. La escuela no me aceptó por ser una ciudad pequeña, ahí fue un choque (Entrevista D, mujer transexual).

Bueno, mi vivencia en la escuela, yo era una persona muy comunicativa, muy amiga de las personas, entonces como se dice, hice del limón una limonada (Entrevistada B, mujer transexual).

Si por un lado la violencia aparentemente no existe en la escuela (o este fenómeno es negado en esta institución), lo que se percibe es que ella está presente y, para sobrevivir en ella, los sujetos acaban encontrando salidas para enfrentamiento, a veces negando o acostumbrándose con la violencia, o 'transformando un limón en una limonada' (SIC, entrevistada B), o sea, convive con la violencia y encuentra formas hasta caricaturizadas al extremo para atraer el afecto de los sujetos presentes. 
Moscovici (2012) al analizar situaciones que demandan que los sujetos produzcan formas de acción para el afrontamiento, indica el concepto de presión a la inferencia. Ribeiro (2017) señala que ante la violencia no son raras las ocasiones en que los sujetos la representan como algo común, natural, soportándola como única salida para vivir.

Con relación a la institución familia, está representada en esta clase por la indicación a las palabras 'familia', 'madre', 'padre', 'hermano' y 'amigo'. En estas, la importancia de la familia en el proceso de aceptación dice de la presencia o no de facilidades de enfrentamiento a las violencias, así como, puede ser motor para la existencia de estas.

Mira, en realidad esta aceptación de ser una transexual es un poco complicada, porque mi madre biológica nunca aceptó por ser homosexual, ya mi madre adoptiva siempre me aceptó. [...] la familia al principio no cree, cree que es una locura (Entrevistada B, mujer transexual).

Mi familia, gracias a Dios, nadie tuvo preconcepto, solo que acogerme también nadie me acogió, ¿es una cosa irónica no? (Entrevistada G, travesti, transfeminina, no binaria).

Bueno, en el caso tipo, así, yo soy una persona bendecida, porque en mi casa cuando me senté para hablar de transexualidad con mi familia, fue con unos 19 años, yo no entendía [...] (Entrevistada E, mujer transexual).

Usted tiene que luchar por lo que usted quiere, podría haber seguido todos los consejos de mi padre y mi madre. Ellos nunca hablaron no conmigo, solo se sentaban. Todo lo que iba a hacer mi padre: ¿vamos a sentar, vamos a hablar? (Entrevistada A, mujer transexual).

Se nota que tanto los sujetos entrevistados como los sujetos con los que estos convivieron a lo largo de sus vidas que estos están elaborando sus representaciones sociales sobre lo que es la transexualidad y el trasvestismo. Como ya se ha mencionado, ante un objeto o fenómeno considerado 'nuevo' o 'extraño', los sujetos pueden producir representaciones sociales que lo asimilan, niegan o asumen totalmente (Ribeiro, Carvalho \& Antunes-Rocha, 2017). Se nota también que las reacciones 
ante fenómenos y objetos nuevos o extraños también pueden generar reacciones de violencia, que están relacionadas incluso con una representación social de negación del mismo.

Por otro lado, la familia de la persona transgénero también es cuestionada por las otras instituciones sobre la orientación sexual e identidad de género de los hijos. Hay casos en que la escuela no acepta el proceso transicional que vive el niño / adolescente, haciendo que el sujeto sea expulsado o, por la insistencia, abandone la escuela. Así, una entrevistada relató: "La directora de la escuela siempre llamaba a mi madre y mi padre: aquí, hay algo mal con su hijo, él no quiere hacer educación física, no quiere jugar fútbol [...]" (Entrevistada A, mujer transexual). Si por un lado la familia y la escuela son instituciones que marcan la trayectoria transicional de las personas entrevistadas, por otro lado, se puede inferir que estas mismas instituciones son responsables de, en el proceso de transición, ser o no elemento generador de violencias debido a sus representaciones sociales sobre el objeto analizado. Una familia que acoge, orienta y busca, a pesar de muchas dudas que puedan surgir, entender lo que pasa con el sujeto transgénero, así como una escuela que desarrolla el respeto la diversidad, promueve el protagonismo y erradica el preconcepto, son esenciales para un cambio cultural y de la sociedad en respeto a las diferentes identidades de género y sexualidades.

\section{Vivencias de la violencia}

Esta clase fue denominada vivencias de la violencia, constituida por el 25\% de los datos, teniendo elementos de cómo la violencia es vivida, percibida y sentida en diferentes ambientes y ocasiones, o aún las prácticas de violencias y las reacciones de los sujetos. Las expresiones 'asî', 'nombre', 'percibir', 'respeto', 'preparar', 'registro', 'nombre social', 'incomodo', 'violencia', 'preconcepto', entre otras son las más frecuentes en esta clase. Se hace interesante remarcar que gran parte de los trechos de esta clase dicen de aquellos establecidos anteriormente como pertenecientes al acceso y utilización de los servicios públicos de salud y 
educación y por ello indican anclajes y objetivaciones en torno al no respeto al uso del nombre social y al preconcepto.

Así, además del proceso de asumir la transexualidad, esta clase trae en sí como la violencia contra los sujetos transgéneros se hace desde estructuras más elementales, como la enunciación del nombre jurídico en lugar del nombre social. Esta violencia, muchas veces cometida con o sin motivación tiene dos caras, deslegitima la identidad al mismo tiempo que proporciona incomodidad e insatisfacción.

Mira, usted no es tratada como una persona normal mientras usted no tiene en su registro [RG] (Entrevistada E, mujer transexual)

Es necesario lidiar con el preconcepto: lidiar con el nombre que está en el RG, lidiar con el cuerpo (Entrevistado I, hombre transexual no binario).

Usted llega a estos ambientes está su nombre social allí, pero la persona todavía llama por el nombre de registro es por provocación porque no tiene otro motivo (Entrevistado D, hombre transexual).

Dependiendo del profesional como la gente bien dijo, si no tiene preparación usted va a sufrir algún tipo de incomodidad sea en el llamar el nombre, o sea en la forma de tratamiento (Entrevistada E, mujer transexual).

El direccionamiento a la actuación de los profesionales de la salud dice de la vivencia que se repite en diferentes casos en que la identidad de género incorporada en la utilización de un nombre social no es respetada. Esto sucede en la actuación profesional y puede revelar por un lado la falta de preparación, además de la ausencia de utilización de conductas en respeto a la población travesti y transexual y por otro lado la existencia de RS sobre la transexualidad que son dispares entre los usuarios y los profesionales de las políticas pública. Aunque existen errores de formación, una de las entrevistadas da pistas para entender cómo los procesos sociales estructurantes auxilian en la consolidación de RS que inciden en preconcepto y violencia: 
La sociedad es muy machista, queriendo o no incluso muchas mujeres son machistas y dependiendo del ciclo del trabajo es, queriendo o no, algún tipo de machismo impera y cuando es en una situación de esas usted percibe cómo el machismo es sofocante (Entrevistada E, mujer transexual).

Sin embargo, otra entrevistada afirma que es una cuestión de percepción, que el uso del nombre social es un síntoma de un sistema cargado de preconceptos-representaciones sociales, aunque ella tenga esperanza de que sea posible concientizar, cambiar la situación, hacer percibir.

Pero cuando ellas, tipo así, perciben la noción de eso, ellas se van a policial más cuidado, más respeto, ¿sabe? (Entrevistada E, mujer transexual).

Ribeiro y Antunes-Rocha (2018) indican que las RS son susceptibles de sufrir alteraciones a lo largo del tiempo, eso si los procesos sociales son cambios en las formas de pensar, sentir y actuar hacia cambios en la información, contextos y actitudes. Ribeiro (2019) complementa que cambios en las identidades y en las formas de los sujetos también pueden indicar movimientos en las RS. En este camino, la cuestión del respeto no puede ser tratada como algo natural, aunque existan procesos sociales que anteceden a las RS, siendo necesaria la concientización. Por otro lado, la falta de respeto puede ser combatida, por medios jurídicos y legislativos necesarios para incitar al cambio social.

Aquí porque tú eres loca, sabe no hay un proceso de respeto natural. El respeto no viene de forma natural él tiene que venir por el miedo tipo no te metas con aquel maricón no que él está loco más o menos así (Entrevistada G, travesti, transfeminina, no binaria).

Creo que deberían primeramente ser atendidas como seres humanos con respeto entendió independiente de la situación sexual, porque no es opción, opción es cuando usted puede optar por una cosa (Entrevistada B, mujer transexual). 
Bueno yo quería a respecto de la violencia que está existiendo ahora, esta cantidad de muerte de transexuales y la policía debería se empeñar más verdad, usted ve que la mayoría de los casos que ocurrieron este año ninguno fue resuelto aún (Entrevistada $A$, mujer transexual).

Si entonces es ahí yo creo así muchas cosas tipo así que la gente sufre así de violencia y es constante esa falta de respeto en cuestión de su género (Entrevistada $\mathrm{E}$, mujer transexual).

En este sentido, la vivencia de la violencia deja huellas físicas, psicológicas y sociales, notadas en la propia enunciación de la palabra 'violencia', indicativo que existen representaciones sociales de este fenómeno. Sin embargo, a pesar de algo indeseado la violencia es vivenciada todos los días en diversos espacios y ocasiones. Aquí hay nuevamente las indicaciones de anclajes y objetivaciones: la violencia es tratada como algo incómodo, de impedimento de vida.

No voy a hablar violencia, creo que es una palabra muy pesada, pero genera sí, una molestia tan psicológica para usted (Entrevistada E, mujer transexual).

[violencia es] cualquier forma de opresión, cuando quieren te oprimir, cuando no quieren dejar que usted sea quien usted es, eso ya es una violencia, y es la mayor de las violencias, por qué es la mayor prisión sin muros. [...] Nosotros sufrimos violencia todos los días. Como ellos hacen, lo que ellos hacen para ayudarnos, eso no tiene ayuda (Entrevistada C, mujer transexual).

Yo recuerdo en varios eventos ya pasó de las personas en el medio de la meriende coger un plato de comida y tirarlo en el medio de mi cara e, así, eso era visto en la escuela apenas como una violencia entre adolescentes, porque tuvo una riñita allí a la hora en la cantina, ¿sabes? (Entrevistada G, travesti, transfeminina, no-binária).

De esta forma, esa clase dice de cómo las experiencias sociales auxilian en la construcción de la consideración de las representaciones sociales de la violencia, principalmente indicando anclajes y objetivos para tal fenómeno. Lo que se percibe es que en la trayectoria de vida 
de cada uno de los participantes los hechos que pueden ser calificados como violencia son muchos, cotidianos, naturalizados, y que el propio sujeto lo indica así, a pesar de sentirlo. El lugar de lo vivido y experimentado es marcado en la consolidación de representaciones sociales (Jodelet, 2005), lo que puede incidir en dificultades mayores para la superación de situaciones estigmatizantes y excluyentes, ya que los propios sujetos que la sufren pueden verla como 'normal' o 'natural'.

\section{Cuerpo y vivencia social}

Por su parte, la clase denominada por datos y vivencia social, constituida por el $28.2 \%$ de los datos. Esta clase demuestra cómo el cuerpo la persona transexual y travesti es un elemento destacado de la forma en que la sociabilidad es vivenciada y en cómo las RS de la violencia son construidas por estos sujetos. Esta clase tiene gran parte de su composición por la categoría preestablecida como rediseño sexual, es decir, fragmentos en que el / la participante dice del deseo o no, de hacer cirugías de 'adecuación' de los genitales. De esta forma, la transformación del cuerpo puede ser lo que trae los elementos de la transexualidad y travestismo a la superficie, hace asumir y aceptar, así como es elemento de discriminación y producción de la violencia. Como ya se ha mencionado sobre las representaciones sociales, según Ribeiro y Antunes-Rocha (2018) y Moliner (2001), parten de cambios en el contexto, en las actitudes y en las informaciones, así como en la identidad (Ribeiro, 2019). En este sentido, los propios cambios en el cuerpo, en la forma de verse y en los contextos e informaciones que el sujeto transgénero pasa a recibir y percibir pueden indicar movimientos también en sus RS.

Por otro lado, es el cuerpo de la/el transexual que realza su identidad de género, además de que es el cuerpo, la marca física de la personalidad, es que se hace docilitar por las transformaciones, cirugías, implantes, procedimientos estéticos, uso de hormonas. Así como es el mismo cuerpo que sufre la violencia, que es descuartizado, violado y manipulado. También existen aquellas personas que se oponen, que se resisten y que enfrentan el sistema binario de género se configura a través de las posibilidades vaginas-mujeres-femeninas y hombres-pene-masculinos 
(Bento, 2008), o aún, el hombre y el masculino pueden fácilmente significar un cuerpo femenino o masculino y viceversa (Butler, 2017).

Así, es en esta clase que las palabras 'travesti', 'cuerpo', 'sociedad', 'morir', 'visibilidad', 'hormona', 'programa' y 'trabajo' son más representativas. Como ya se ha dicho, en esta correlación es posible ver cuánto el travestismo y la transexualidad están ligadas a las cuestiones corporales, estas a su vez también atraviesan la RS de violencia. Al enunciar la palabra 'travesti' hay la demarcación de las diferencias en relación a la aceptación, reconocimiento y violencias que distinguen a las personas trans de las travestis. Si estas identidades son confundidas socialmente, las formas de tratamiento también son confusas, lo que puede generar disparidades en la aceptación y generar aún una patologización de una identidad.

Estoy viendo a las personas poniendo bandera transexual, haciendo tipo revuelo en la Internet, haciendo manifestación [en relación con la aceptación de la transexualidad], porque para travesti que no tienen acceso al nombre social es un desconocimiento y marginalidad que rueda mucho e incluso borrado (Entrevistado I, hombre transexual no binario).

Ser travesti es una forma de resistencia en lo que yo digo es la lucha diaria por la supervivencia, no es glamour como la gente dice (Entrevistada F, mujer transexual).

Pero ellos están siempre queriendo saber, con la excusa de que necesitan entender y también vinculado la cuestión de que si ella probablemente es travesti, ella tiene disforia de género, ella está loca, ¿sabe? (Entrevistada G, travesti, transfeminina, no binaria).

En un segundo momento de esa clase es posible identificar la relevancia de la expresión "cuerpo" como una articuladora de la RS de la violencia, siendo este el que lo/la diferencia y aquel también recibe la violencia. Hay la correlación con la aceptación y diferenciación de lo que es o no ser trans. Hay en estos intentos de definir lo que es ser transexual o travesti a partir de la relación con el cuerpo (estar de acuerdo lo físico y lo psicológico o no). 
Es el cuerpo de ella, ella tiene derecho! Creo que aunque la hormona hiciera mal es de pura responsabilidad de la persona con su cuerpo (Entrevistada G, travesti, transfeminina, no binaria).

Si por ejemplo usted es una nińa que nació en un cuerpo de niño y ahora usted asumió como mujer usted tiene que ser tratada tipo así en lo mínimo como tal y listo (Entrevistada E, mujer transexual).

La cuestión transexual es la misma cosa, ya es psicológica, la trans ella tiene la necesidad porque ella no ve el cuerpo de ella un cuerpo que ella siente cómodo (Entrevistada G, travesti, transfeminina, no binaria).

Lo que se percibe de esas conversaciones es que el elemento 'cuerpo' es un articulador de la RS de la violencia, pudiendo ser considerado ahora como objetivación (aquel que define lo que es el sujeto transgénero) ahora como anclaje (es el cuerpo que es el incitador de la violencia). Se nota también que hay un resquicio del pensamiento social de la necesidad de adecuación del cuerpo, de los genitales, principalmente, a la identidad del individuo. Sin embargo, como ya se ha afirmado, hay que cuestionar la necesidad de una cirugía para garantizar la cura o la felicidad ante un cuerpo que cuestiona el sujeto y también a la sociedad binaria.

El anclaje/objetivación en los datos de la RS de la violencia también es notado en el sentido de la adecuación del cuerpo a las necesidades y deseos de la persona que se identifica como travesti y transexual hay la expresión 'hormona' y 'hormonización'. Siendo una técnica presente tanto para hombres o mujeres trans y travestis, ella dice de la eliminación/ganancias de caracteres físicos como barba, voz, etc. Hay en la conversación de los participantes la preocupación, ante la hormonización si esta práctica puede hacer bien o mal.

Chicas, niñas pequeñas, ya tomando hormona porque ya entraron en el proceso de la adolescencia, en la pubertad. [...] Es el cuerpo de ella y ella tiene derecho, entonces la gente sabe que la hormona no hace dańo desde que sea controlado por un endocrino. [...] Algunas toman cualquier tipo de hormona y tienen elefantiasis, trombosis, AVC, derrame, porque no tiene acceso. Así si una persona que tiene 
hambre no tiene acceso a trabajo y comida (Entrevistada G, travesti, transfeminina, no binaria).

[los médicos] no entiende nada de silicona, de hormona. Es porque son omisos incluso porque no quieren tanto, es que en toda facultad de medicina la mayor que yo sé ellos no está ni ahí para transexual, ellos estudian más el órgano genital (Entrevistada F, mujer transexual).

Mismo que es una práctica corriente a la población trans, se debe cuestionar el acceso a la hormonoterapia, ya que no hay muchos registros de la atención a esta especificidad en el Sistema Único de Salud, mientras que el acceso a profesionales habilitados y preparados es precario o, a veces sucede con muchos cuestionamientos, discriminación y preconcepto. En muchos casos los niños y las niñas trans optan por buscar en la clandestinidad el acceso a la hormonización, usándolos sin prescripción médica y evaluación de impacto para su salud.

¿Este intento de convertirse sería una prerrogativa de atender a las expectativas de la sociedad y cesar con la violencia? ¿O un intento de incluirse por la estandarización esperada? ¿Corresponde a las demás representaciones sociales de hombres y mujeres? La presencia de la expresión «morir` puede ayudarnos en el entendimiento de esa corrección sociedad - cuerpo [de las personas travestis y transexuales] - violencia.

Actualmente hago terapia, exactamente sobre esa cuestión, para poder aceptar este cuerpo en el cual vivo [...] porque llegué a la conclusión: es ese cuerpo que yo voy a habitar el resto de mi vida (Entrevistado I, hombre transexual no binario).

[sobre la violencia que sufrió] Mis amigos y mi novio van a entrar en el medio, son personas así que van a sufrir y pueden incluso morir por una cosa que me pasó, si yo estuviera sola, sería otro rollo (Entrevistada G, travesti, transfeminina, no binaria).

Se alojó en la parte que quedaba la silicona, allí dio necrosis en aquel lugar donde la bacteria se alojó, ahí tuve que drenar toda la silicona, la parte que murió ele tuvo que quitar" (Entrevistada A, mujer transexual). 
En esta clase se percibe cómo entender el elemento cuerpo es esencial para comprender el fenómeno de la violencia en las poblaciones travestis y transexuales y sus representaciones sociales. El cuerpo es el receptáculo de la violencia del otro, pero también es aquel que necesita ser modificado,a veces, para la adecuación. Posicionarse contra la necesidad de rediseño es de alguna manera no colocar la violencia como algo natural, es otra forma de ponerse sobre la experiencia, resistiendo ante la violencia sufrida y representada.

\section{Procesos identitarios}

Por último, la clase denominada identidad y género fue constituida por el $14.78 \%$ de las palabras utilizadas para el análisis. En ella, es posible percibir la relación entre la violencia con las cuestiones identitarias, con lo diferente. Por tanto, las expresiones más frecuentes en esta clase fueron: 'hombre', 'cisgénero', 'género', 'mujer trans', 'cis', 'hombre trans', 'identificar', 'mujer', etc. Aquí vemos que la violencia, en términos representacionales, es aquella demarcada por la no identificación y de la negación del derecho de ser quienes son. Así, están representadas en esa clase la necesidad de diferenciación y de definición lo que es ser una persona travesti/transexual, trayendo lo que es ser transgénero en oposición a lo que es ser cisgénero. En ese recorrido queda anclada a la violencia la disposición de las diferencias, principalmente aquellas relacionadas a las violencias que las personas transexuales/travestis sufren por tener una identidad de género discrepante a la cisgeneridad.

En el medio LGBT creo que no porque se oye que el hombre gay, porque el hombre gay tiene privilegios, después de todo es un hombre (Entrevistado I, hombre transexual no binario).

Si nadie nunca llegara a la cabeza de un nińo que probablemente es un niño transgénero y hablara para ella que el cuerpo de una mujer es así y el cuerpo de un hombre es así, iba crecer viendo lo que tiene entre las piernas es incorrecto (Entrevistada G, travesti, transfemenina, no binaria). 
El servicio de salud ya es difícil incluso para una persona que es un hombre o una mujer, digamos así que heteronormativa, imagina para una transexual, para una trans, para una travesti (Entrevistada $\mathrm{B}$, mujer transexual).

Si tiene dos personas, dos hombres, dos mujeres, si una es transexual, gay, se ríen en su cara entendió (Entrevistada B, mujer transexual).

Estas conversaciones demuestran que existen jerarquías dentro de la propia comunidad LGBT, lo que puede indicar que estas tienen relación con las violencias que sufren cada uno de los sujetos con identidades de género y orientaciones sexuales diferentes de lo que es el cisgénero o heterosexuales. A partir de estos trechos, también es posible notar que la violencia está desde la imposición de la identidad de lo que es ser hombre o mujer hasta la diferenciación en la convivencia social, en las escenas de preconcepto, transfobia y exclusión por no ser cisgénero. Hay en esta correlación una contribución para el entendimiento de la RS de violencia para los sujetos entrevistados, además de la mediación del cuerpo, ella está íntimamente relacionada (anclada) con la forma en que la identidad es asumida, diferenciada y aceptada.

\section{Consideraciones finales}

El análisis de las representaciones sociales de la violencia hacia sujetos transexuales suscita la comprensión que las vivencias marcan las formas de pensar, sentir y actuar de esos sujetos. Siendo desde el proceso de asumir la transexualidad/travestilidad para sí o para la familia, instituciones sociales y actores sociales, esta no sucede de forma sin resistencias, sin violencia.

El elemento 'cuerpo' permite anclajes y objetivaciones mediando la representación social de la violencia para sujetos travestis y transexuales. El cuerpo media, porque es en el cuerpo que se siente las principales violencias, es debido al cuerpo que no se adecua a un patrón binario, cis-hetero-normativo que las violencias ocurren, es el cuerpo que demanda intervención mediante él que sucede la relación social. 
Con la clase tres se verifica cuanto la RS de violencia también está relacionada con los procesos de diferenciación e identificación, siendo posible entender a partir de los entrevistados que es justamente por eso que la violencia ocurre.

No se puede negar el tema de la violencia contra la población transgénero ha provocado a la sociedad a posicionarse y eso interfiere directamente en la construcción y el mantenimiento de las representaciones sociales. Este estudio muestra las RS a partir de los sujetos que están sufriendo tales violencias, enfrentando cotidianamente los cambios sociales.

Como se afirma a lo largo del análisis, en la representación social de la violencia se percibe que hay restos de lo que se ha divulgado en los medios sobre la cuestión de la transexualidad y el travestismo. La visualización de la obligación de la "cirugía de cambio de sexo" da aires de estandarización a la población trans, o sea, hay una enfermedad que hay que tratarla con una cirugía, sin embargo, se sabe que esta identidad extrapola las cuestiones corporales.

En ese sentido, aunque el proceso de transición esté marcado por los cambios en el cuerpo se cree que la principal transición vivida por los sujetos transgéneros esté en su relación con lo social. En realidad, existe una relación casi cíclica entre esas dos transiciones, al cuerpo de la persona travesti y transexual es normatizado por la vivencia social, o, en algunos casos, es utilizado como elemento de resistencia a las presiones sociales. Sin embargo, estando docilitado / estandarizado en una forma más aceptable o siendo acto de resistencia, él es víctima por el preconcepto, la discriminación y las agresiones. Esconder la sexualidad y la identidad es una forma de ser menos víctima de violencias en la calle, en el trabajo y en casa.

Sutil, pero presente, la violencia contra las personas trans por parte de los profesionales de las áreas de la salud y de la educación se hicieron notar. Aunque existan buenas intenciones y profesionales atentos a las cuestiones de identidad de género, todavía hay un largo camino por recorrer, que pasa desde la concientización durante los trayectos formativos, desmitifica y desproblematiza la cuestión, hasta la propuesta 
e implantación de políticas públicas más ásperas en lo que se refiere a la tolerancia a las diferencias, a la diversidad y a la atención, a las especificidades de la población travesti y transexual. Este camino de las políticas públicas debe acompañar el cambio de la sociedad y de la cultura.

Este estudio presenta limitaciones por traer solo nueve sujetos en su composición y análisis, no en tanto demuestra lo cuánto hay un campo provechoso de investigaciones a ser hechas. No solo en el umbral de entender lo que las personas piensan sobre la transexualidad o el travestismo, sino estudios que coloquen a la población transexual y travesti como sujetos y participantes activos de sus procesos.

\section{Referencias}

Antunes-Rocha, M., Amorim-Silva, K., Benfica, W., Carvalho, C. \& Ribeiro, L. (2015). Representaçóes Sociais em Movimento: desafios para elaborar o estranho em familiar. XII Congresso Nacional de Psicologia Escolar e Educacional of 37th Annual Conference of the International Scholl Psychology Association (pp. 836-841). São Paulo.

Arán, M., Murta, D. \& Lionço, T. (2009). Transexualidade e saúde pública no Brasil. Ciência \& Saúde Coletiva, 14(4), pp. 11411149. http://dx.doi.org/10.1590/S1413-81232009000400020

Arbatani, T., Aqili, S., Labafi, S. \& Omidi, A. (2016). Social representations of iranian transsexual people in the media: a thematic analysis. International Journal of Academic Research in Business and Social Sciences, 6(5), 273-284. https://doi.org/10.6007/ IJARBSS/v6-i5/2142

Assis, C. (2013). "Entre tapas e beijos": representaçóes sociais sobre a violência de gênero para adolescentes. Psicologia e saber social, 2(2), 229-242. https://doi.org/10.12957/psi.saber.soc.2013.8796

Baptista, G., Silva, O., Menandro, M. \& Bonomo, M. (2016). Representaçóes sociais de homens e mulheres transexuais para estudantes 
de psicologia. Anais do IV Seminário Internacional de Educação e Sexualidade (pp. 1-15). Vitória-ES. Recuperado de http:// www.gepsexualidades.com.br/resources/anais/6/1466680421_ ARQUIVO_Pessoastranstrabalhocompleto(ultimaversao).pdf

Bento, B. (2008). O que é transexualidade. São Paulo: Brasiliense.

Brasil. (2013). PORTARIA $N^{\circ}$ 2.803, DE 19 DE NOVEMBRO DE 2013 - Redefine e amplia o Processo Transexualizador no Sistema Único de Saúde (SUS). Brasília: Ministério da Saúde. Recuperado de http://bvsms.saude.gov.br/bvs/saudelegis/gm/2013/ prt2803_19_11_2013.html

Butler, J. (2017). Problemas de gênero: feminismo e subversão da identidade (13a ed.). Río de Janeiro: Civilização Brasileira.

Camargo, B. \& Justo, A. (2013). Tutorial para uso do software de análise textual IRAMUTEQ. Florianópolis: Laboratório de Psicologia Social da Comunicação e Cognição (LACCOS) - UFSC. Recuperado de www.iramuteq.org/documentation/fichiers/ tutoriel-en-portugais

Caravaca-Morera, J. \& Padilha, M. (2017). Social representations of sex and gender among trans people. Revista Brasileira de Enfermagem, 70(6), 1235-1243. http://dx.doi.org/10.1590/ 0034-7167-2016-0581

Carrieri, A., Souza, E. \& Aguiar, A. (2014). Trabalho, Violência e Sexualidade: Estudo de Lésbicas, Travestis e Transexuais. $R A C$ Revista de Administração Contemporânea, 18(1), 78-95. https:// doi.org/10.1590/S1415-65552014000100006

Correia, C. (2014). Representaçóes sobre o suicídio para mulheres com história de violência doméstica e tentativa do mesmo. Texto Contexto - Enfermagem, 23(1), 118-125.

Cortez, M., Menandro, M. \& Souza, L. (2010). Representaçóes sociais da violência de gênero/conjugal em estudos científicos. Anais do Fazendo Gênero 9: diásporas, diversidades, deslocamentos. Florianópolis: UFSC. Recuperado de http://www.fazendogenero.ufsc. br/9/resources/anais/1267363401_ARQUIVO_FG9Cortez.pdf 
Cruz, E. F. (2011). Banheiros, travestis, relações de gênero e diferenças no cotidiano da escola. Revista Psicologia Politica, 21(11), 73-90.

Efrem Filho, R. (2016). Corpos brutalizados: conflitos e materializaçôes nas mortes de LGBT. Cadernos Pagu(46), 311-340. http://dx.doi.org/10.1590/18094449201600460311

Guimarães, S. P. \& Campos, P. F. (2007). Norma social violenta: um estudo da representação social da violência em adolescentes. Psicol. Reflex. Crit., 20(2), 188-196. https://doi.org/10.1590/ S0102-79722007000200003

Itaqui, L. \& Felippi, G. (2017). Cinema, psicologia e homoafetividade: reflexões das cenas do contemporâneo. En L. P. Ribeiro, Familia(s) e afetividade(s) (pp. 131-146). Río de Janeiro: Gramma.

Jodelet, D. (2001). As representaçôes sociais. Rio de Janeiro: EdUERJ. Jodelet, D. (2001). Representações sociais: um domínio em expansão. En D. Jodelet, As representaçóes sociais (pp. 17-44). Río de Janeiro: EdUERJ.

Jodelet, D. (2005). Experiência e representaçóes sociais. En M.S. Menin \& A. d. Shimizu, Experiência e representação social: questóes teóricas e metodológicas (pp. 23-56). São Paulo: Casa do Psicólogo.

Lanz, L. (2014). O corpo da roupa: a pessoa transgênera entre a transgressáo e a conformidade com as normas de gênero. Programa de Pós-Graduação em Sociologia, Departamento de Ciências Sociais. Curitiba: Universidade Federal do Paraná.

Matão, M., Miranda, D., Campos, P., Teles, M. \& Mesquita, R. (2010). Representaçóes sociais da transexualidade: perspectiva dos acadêmicos de enfermagem e medicina. Revista Baiana de Saúde Pública, 34(1), 101-118. Recuperado de http://files.bvs. br/upload/S/0100-0233/2010/v34n1/a1423.pdf

Moliner, P. (2001). Introduction. En P. Moliner, La dynamique des représentations sociales: pouquoi et comment les représentations se transforment-elles? (pp. 7-14). Grenoble: Presses Universitaires de Grenoble (PUG). 
Moscovici, S. (2012). A psicanálise, sua imagem e seu público. Petrópolis/RJ: Vozes.

Oliveira, A., Chamon, E. \& Maurício, A. (2010). Representação social da violência: estudo exploratório com estudantes de uma universidade do interior do estado de São Paulo. Revista Educar, 261-274. https://doi.org/10.1590/S0104-40602010000100017

Porto, M. (1999). A violência urbana e suas representaçóes sociais: o caso do Distrito Federal. São Paulo Perspectiva, 13(4), 130-135. https://doi.org/10.1590/S0102-88391999000400014

Ribeiro, L. (2019). IDENTIDADE E REPRESENTAÇÓES SOCIAIS: evidências e correlaçóes a partir de pesquisas da área da Educação. Relatório de Residência Pós-Doutoral, Universidade Federal de Minas Gerais, Programa de Pós-Graduação em Educação: Conhecimento e Inclusão Social, Belo Horizonte.

Ribeiro, L. P. (2017). O campo, a violência e a educação do campo: representaçóes sociais sobre a violência de educandos do curso de licenciatura em educação do campo. Río de Janeiro: Gramma.

Ribeiro, L. P. \& Maria, I. A.-R. (2018). Representaçóes Sociais em movimento: pesquisas em contextos educativos geradores de mudança. Curitiba: Appris.

Ribeiro, L. \& Antunes-Rocha, M. (2016). História, abordagens, métodos e perspectivas da teoria das representaçóes sociais. Psicologia e Sociedade, 28(2), 407-409. http://dx.doi.org/10. 1590/1807-03102016v28n2p407

Ribeiro, L., Carvalho, C. \& Antunes-Rocha, M. (2017). Representaçóes Sociais em Movimento: uma análise de duas pesquisas no âmbito da Educaçáo do Campo da FaE-UFMG. Educação e Cultura Contemporânea, 14, 343-366. https://doi.org/ $10.5935 / 2238-1279.20170052$

Rocha, A., Ban, G. \& Valenga, R. (21 de agosto de 2017). Brasil é $o$ pais que mais mata travestis e transexuais no mundo. Periódico UEPG. Recuperado de http://periodico.jor.br/index.php/ direitos-humanos/669-brasil-e-o-pais-que-mais-mata-travestise-transexuais-no-mundo 
Santos, A. B., Shimizu, H. E. \& Merchan-Hamann, E. (2014). Processo de formação das representações sociais sobre transexualidade dos profissionais de saúde: possíveis caminhos para superação do preconceito. Ciência e Saúde Coletiva, 19(11), 4545-4554. http://dx.doi.org/10.1590/1413-812320141911.15702013.

Silva, G., Souza, E., Sena, R., Moura, I., Sobreira, M. \& Miranda, F. (2016). Situações de violência contra travestis e transexuais em um município do nordeste brasileiro. Revista Gaúcha de Enfermagem, 37(2), 1-7. https://doi.org/10.1590/1983-1447.2016.02.56407

Souza, H. \& Bernardo, M. (2014). Transexualidade: as consequências do preconceito escolar para a vida profissional. Bagoas - estudos gays: gêneros e sexualidades, 8(11), 157-175. Recuperado el 25 de noviembre de 2017 de https://periodicos.ufrn.br/bagoas/article/ view/6548/5078

Transgender Europe. (30 de marzo de 2017). TMM Update Trans Day of Visibility 2017 - Press Release. Transgender Europe. Recuperado de http://transrespect.org/en/tdov-2017-tmm-update/

Ventura, M. (2010). A transexualidade no tribunal: saúde e cidadania. Río de Janeiro: EdUERJ.

Zanin, H., Ferreira, L. \& Ribeiro, L. (2019). Ingresso e Permanência no Trabalho e Empregopor Sujeitos LGBT+ em Belo Horizonte, Brasil. Id on line: revista multidisciplinar e de psicologia, 13(44), 460-474. https://doi.org/10.14295/idonline.v13i44.1624

Recibido: 9 de abril, 2018 Revisado: 18 de febrero, 2019 Aceptado: 5 de marzo, 2019 